\title{
OPTIMIZATION OF HORIZONTAL HIGHWAY ALIGNMENT USING A PATH PLANNER METHOD
}

\author{
SUSHMA MB \& AVIJIT MAJI \\ Indian Institute of Technology Bombay, India
}

\begin{abstract}
This paper proposes Path Planner Method (PPM) that operates on the principal of Rapidly-exploringRandom Tree (RRT) algorithm to obtain an optimal horizontal alignment. It has the ability to efficiently search in non-convex high dimension space. It primary goal is to explore the entire study area by generating random point of intersections (PIs). In this, a tree like path is developed iteratively by expanding from start to end point. These paths would meet the requirements of highway geometric guidelines and minimizes cost. It's an interactive two-stage process. Firstly, it finds an optimum set of PI's connected by tangent sections. Secondly, appropriate curves are fitted at each PI to obtain horizontal alignment. The alignment is checked for encroachment to the restricted area and any violation leads to further refinement of the PI locations. The method's efficiency is verified through a case study using a geographical map of a place in Odisha, India.
\end{abstract}

Keywords: highway design, optimization models, horizontal alignment, PPM.

\section{INTRODUCTION}

In highway alignment design, engineers design the horizontal alignment first, and then develop the vertical alignment according to the horizontal alignment. Well-designed horizontal highway alignment helps to avoid environmentally preserved zones such as forests, wetlands, historic or heritage sites etc. It also provides better connectivity to the intermediate cities, and other important places. Designing optimal horizontal alignment is very challenging as it is constrained by available budget, design specifications, geographical limitations and right-of-way. An infinite number of feasible horizontal alignments may exist between the two connecting destinations. However, selecting the best makes the design process complex. The feasible horizontal alignments are compared based on length dependent cost, location dependent cost and the obligatory points. The obligatory points include control areas through which the alignment need to pass and restricted or prohibited areas that the alignment need to avoid. Any modification in the horizontal alignment can lead to change in overall project cost and impact to the constraints. Moreover, restrictions on the horizontal alignment of a highway mainly come from codal provisions [1]. Hence, exploring the feasible horizontal alignments during planning and selecting the best is a rigorous process. Designing of a highway alignment is done in several basic sequential stages (i.e., planning, detail design, construction, etc.). In the planning stage, the need of a road at a region is identified and out of all the possible feasible connections between the two points, a suitable alternative is selected. This alternative is selected if it satisfies the geometric design standards [1] and minimizes the environmental impact. After selecting a preferred alternative in planning, a detail design is prepared in the design stage. The aim of this stage is to set the complete highway project plan ready for construction in the real world. Traditionally, highway alignment is designed based on the experience of the engineers. Engineers select some alternatives as initial routes and iteratively improve them based on various design constraints. The best alternative with the minimum length and the lowest impact is selected at the end for detail design. This is a manual process and hence, the obtained alignment does not guarantee an optimal solution. Moreover, manual evaluation of all feasible solutions for 
various alignment costs and impacts is time taking and intensive procedure. To overcome this limitation, researchers have developed computer-aided optimization models for horizontal alignment design. These models develop the highway alignment in the planning stage. Generally, in these models, the PIs are generated on regularly spaced fixed orthogonal planes and joined with appropriate tangent and curve sections to form the horizontal alignment. These PI locations are optimized using evolutionary algorithms [2]-[6]. In these models, the PI locations are confined to the orthogonal planes and restricted by its number. This would affect the precision of the generated horizontal highway alignment. These models are also constrained with constant radius value for the horizontal curves. In design stage, these radiuses are eventually adjusted for appropriate values, and thus the cost and impact also changes. Moreover, in a complex environment it becomes very difficult to fix a curve with constant radius. Hence, it is better to accommodate curves with appropriate radius between the tangent sections in the planning stage, so that the effort in the design stage is reduced.

This paper endeavours an optimization model to overcome such limitations. The aim of the model is to bridge the existing gap between highway planning and design stage. The proposed model has no location constraints for developing intermediate PI's and also considers variable value for circular radius. This renders benefit to the existing highway design by increasing the precision of the generated highway alignment in the design stage. This model develops an alignment considering the objectives and guidelines requirements for designing an optimal horizontal highway alignment by exploring the entire study area. A geographic information system (GIS) based map is used in this study, where the start and end points of the alignment are defined. A set of points that are generated randomly in entire search area are considered as the decision variables. Appropriate curves are fitted along the PIs to obtain an alignment.

\section{HORIZONTAL HIGHWAY ALIGNMENT OPTIMIZATION MODELS}

The highway alignment consists of tangent sections connected with curve sections for smooth change in directions. Generally, circular curves are considered in horizontal alignment to maintain the centrifugal force, and prevent vehicle from lateral skid. This can be achieved by providing appropriate curvature and super elevation based on design speed and side friction. The purpose of highway alignment optimization is to develop a mathematical search model to find a global or near global optimal solution based on the total objective cost minimization within the available geometric design constraints.

In the last few decades many automated computer-aided methods are developed that minimize the total highway alignment cost to obtain an optimized alignment. Howard et al. developed optimum curvature principle (OCP), which was derived from the calculus of variations [7]. It considered the cost function as continuous between the two desired points. However, in real world the cost function cannot be continuous, which created a limitation. The model developed by Parker [8], solves the horizontal alignment problem by network optimization model. It formulates the horizontal alignment optimization problem as a network problem and the total alignment cost is calculated by the linear combination of all the costs assigned to each link of the network. The model proposed by Jha [2], [3], Jha and Schonfeld [4], Jong et al. [5] and Kang et al. [9], considers intermediate PIs at predefined orthogonal planes and fixed radius curves. Mondal [10], developed a model to optimize horizontal alignment along specific corridors. The model used existing alignment as the base alignment and determines the improved horizontal alignment by adjusting the PIs of the existing alignment. The relevant methods and corresponding references are summarized in Table 1. 
Table 1: Optimization models for horizontal alignment design.

\begin{tabular}{|l|l|}
\hline Methods & References \\
\hline Calculus of variation & $\begin{array}{l}\text { Howard et al. [7], Shaw and Howard [11], } \\
\text { Thomson and Sykes [12] and Wan [13]. }\end{array}$ \\
\hline Network optimization & $\begin{array}{l}\text { Trietsch [14], Turner and Miles [15], } \\
\text { Athanassoulis and Calogero [16] and Parker [8]. }\end{array}$ \\
\hline Dynamic programming & Hogan [17] and Nicholson et al. [18] \\
\hline Genetic algorithms & $\begin{array}{l}\text { Jong et al. [5], Jha [2], [3], Jha and Schonfeld [4], } \\
\text { Kang and Jha [9], Tat [19], Maji and Jha [20] and } \\
\text { Maji and Jha [21]. }\end{array}$ \\
\hline Particle Swarm Algorithm & Shafahi and Mehdi [6]. \\
\hline Neighborhood search-heuristic with MIP & Lee et al. [22]. \\
\hline MIP and derivative free optimization & Mondal et al. [10]. \\
\hline
\end{tabular}

\section{HORIZONTAL ALIGNMENT COST}

It is composed of length and location dependent cost. Length dependent depends on the nature of construction and ROW of an alignment associated with a unit length cost. When an alignment is generated it includes length of tangential and curvature section. So, the total alignment length is formulated as in eqn (1) [4], [5]:

$L=\sum_{n=0}^{N-1} \sqrt{\left(x_{n}^{T}-x_{n+1}^{C}\right)^{2}+\left(y_{n}^{T}-y_{n+1}^{C}\right)^{2}}+\sum_{k=1}^{n o s} R_{k} \Delta_{k} \quad$ For all $\mathrm{n}=0,1,2 .(N-1)$,

where,

$L=$ Alignment length

$N=$ Total number of PIs

$\left(x_{n}^{T}, y_{n}^{T}\right)=$ Alignment coordinate at intersection of $n^{\text {th }}$ tangent and previous curve section

$\left(x_{n}^{C}, y_{n}^{C}\right)=$ Alignment coordinate at intersection of $n^{\text {th }}$ tangent and following curve section

$R_{k} \quad=$ Radius of $k^{t h}$ circular curve along the alignment

$\Delta_{k} \quad=$ Intersection angle of $k^{\text {th }}$ circular curve along the alignment

However, the length dependent cost depends on the construction cost, so the total length dependent cost can be evaluated by using eqn (2):

where,

$$
T C_{L N C}=L \times u l d,
$$

$T C_{L N C} \quad=$ Length dependent cost

$L=$ Alignment length

uld = Unit length dependent cost

The location dependent costs are the costs associated with the acquired land to build the highway. It may represent one or several location cost categories such as historical areas, built up areas, etc. Some features that are related to this cost in this study are land acquisitions, and impact on environmental features like wetlands, and forest. Land acquisition cost is associated with the acquired land to build the highway. Total location dependent cost is calculated by counting all the fractional land parcels cost through which the alignment is 
passing. Let, $L_{C U M}$ be the unit cost of the land parcel and $h c_{i}$ the fraction of the area acquired by the highway alignment. Then, the total ROW cost will be $L_{C U M} \times h c_{i}$. If the alignment does not pass through a particular land parcel, then the fraction of area needed from that land parcel will be zero (i.e., $h c_{i}=0$ ). Let the total number of land parcels within the study area be $a p l$, the total location dependent $\operatorname{cost} T C_{L}$ can be estimated as given in eqn (3), [21].

$$
T C_{L O C}=\sum_{i=1}^{a p l} L C U M_{i} \times h c_{i}
$$

where,

$T C_{L O C}=$ Total location dependent cost.

$\operatorname{LCUM}_{i}=$ Unit cost of $i^{\text {th }}$ land parcel in the study area.

$h c_{i}=$ Fractional area of $i^{t h}$ land parcel required for the highway alignment.

\section{OBJECTIVES}

A properly planned highway alignment during planning can save money and time during design. It is possible if some of the critical design steps are incorporated in the planning process like, selecting PI's at right location and fitting curves with appropriate radius. The overall length of the alignment is dependent on the tangent (i.e. determined by PI's location) and the curve section. An appropriate horizontal curve section also helps to minimize the environmental impact. Hence, the overall objective function of the model is to minimize the total cost of the alignment, i.e., by minimizing the weighted sum of the total length of alignment (refer eqn (2)) and impacts on the environmental sensitive areas (refer eqn (3)). Therefore, total alignment $\operatorname{cost}\left(C_{H A}\right)$ is derived as in eqn (4).

$$
\text { minimize } C_{H A}=T C_{L N C}+T C_{L O C}
$$

\section{PATH PLANNER METHOD (PPM)}

In this study, a customized PPM based on RRT [23], [24], is developed to search for an optimal horizontal alignment. The method develops an optimal horizontal alignment in two stages. In the first stage, it attempts to find a good piecewise linear line by selecting the optimal points that satisfies the objective function. In the second stage curves are incorporated to the piecewise linear line, so that a smooth and continuous path is obtained. The two stages in PPM are discussed in the subsequent sections.

\subsection{Stage 1}

Stage one generates a piecewise linear line which approximates the highway alignment. In this approach, tree like path is propagated iteratively from one end location to the other, by joining the random samples in the study area. These, random samples are drawn from the classical sampling theory, dispersion measures, how well a space is covered by a sample set. Given a sample space $X$ and a point set $P \subset X$, The dispersion $\beta$ of $P$ is defined in eqn (5):

$$
\beta(P, \gamma)=\sup _{q \in X} \min _{p \in P} \gamma(q, p),
$$

where, $\gamma$ is a metric on $X$. Intuitively, dispersion is the radius of the largest empty ball (under $\gamma$ ) in the space [25]. The growth of the tree depends on the step size (i.e., the minimum tangent length) based on the number and density of the restricted areas within the study area. The exploration strategy of the method to the unexplored space in the study area is controlled 
(A)

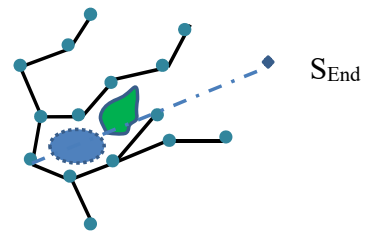

(C)

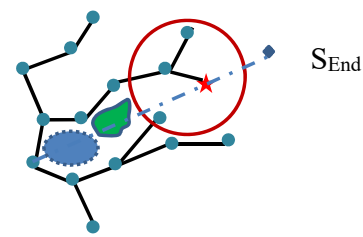

(E)

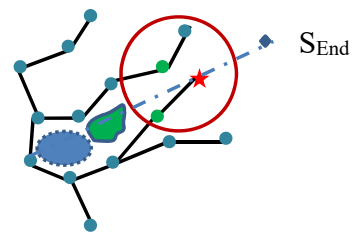

(B)

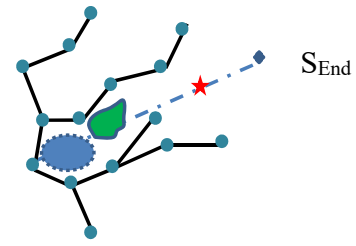

(D)

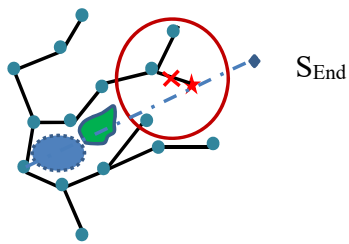

(F)

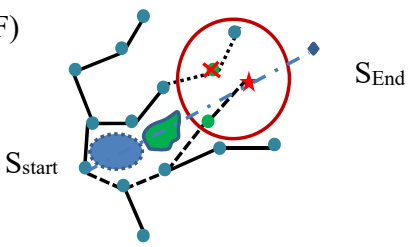

Figure 1: Visualization of the PPM for generation of PIs.

by the Voronoi diagrams [25]. The method first initiates the tree from the start point, $\mathrm{S}_{\text {start }}$, and is constructed by connecting the discretely located random point population within the study area till it reaches the end point, $\mathrm{S}_{\text {End. }}$. A new connection between the available connected points in the tree and a randomly generated point, $S_{\text {rand }}$, is established, only if it possesses the minimum cost for the path connecting the start point, $\mathrm{S}_{\text {start }}$, and satisfies the obligatory point requirements and horizontal alignment design guidelines (i.e., minimum length of the tangential section). The connected points are the feasible PIs of the horizontal alignment. The method incorporates deletion of the points from the tree which does not possess a feasible path, and improves it with generation of more random points. The method searches the entire tree for more optimal connections, and then it reconnects for the refinement of the alignment. The process continues till the termination criteria, is satisfied i.e., either the maximum number of iteration is reached or there is no improvement in the cost of the alignment. This way, the path or the horizontal alignment advances towards the other end location by exploring the study area and connecting the new feasible PIs. Fig. 1 shows the model's visualization for generation of the PIs in PPM respectively.

\subsection{Stage 2}

In highway alignment design both tangent and curve sections are needed to provide a smooth transition. In PPM, various combinations of the radiuses within the allowed minimum and maximum range are selected based on the highway geometric guidelines and the minimum tangent length. Then the radiuses for each PI's are optimized by the sequential quadratic optimization method from which an appropriate curve is selected. The optimization of the curve from the various combinations is based on two criteria: (a) minimize alignment length and (b) should have a minimum impact on the environmentally sensitive areas. These criteria are checked for each curve, and the procedure is repeated until the curve is fitted at all the 
PIs. However, the algorithm terminates for certain condition. If after all the possible combinations of the curve, still the alignment violates any environmental sensitive areas, and then the method terminates to stage 1. In stage 1 a new set of PI's are obtained by generating random points around the section of the alignment where the curve fitting in stage 2 is violated. Fig. 2 shows the flowchart for both the stages in PPM.

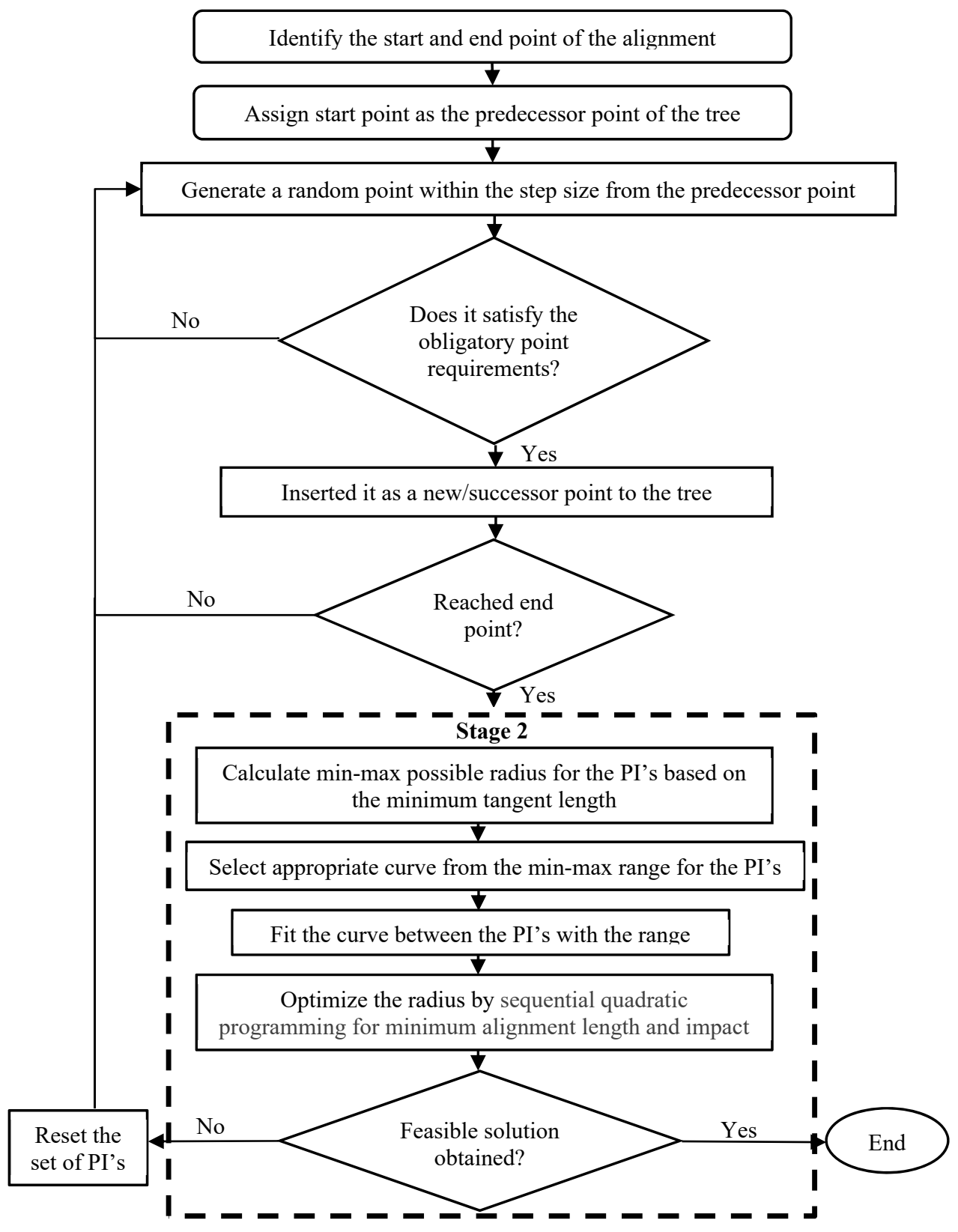

Figure 2: Flowchart for PPM. 


\section{STUDY AREA}

A horizontal highway alignment optimization problem is examined between the two cities, identified as Angul and Talcher in Odisha, India. The lateral and longitudinal coordinate of these cities are $20^{\circ} 51^{\prime} 40.88^{\prime \prime} \mathrm{N}, 85^{\circ} 5^{\prime} 58.84^{\prime \prime} \mathrm{E}$ and $20^{\circ} 57^{\prime} 14.27^{\prime \prime} \mathrm{N}, 85^{\circ} 14^{\prime} 0.48^{\prime \prime} \mathrm{E}$ respectively. In this study Angul is considered as the origin and Talcher as destination with a direct distance of $18 \mathrm{~km}$. The land parcels represented by forest, buildings and land parcels are identified in the selected coordinate of the study area. The land parcels representing wetland and forest areas are considered as environmentally sensitive area. The study area is identified with 14 sensitive areas. These areas are represented together in the alignment optimization process and considered in estimating the environmental impact. Similarly, the historic area and the building areas in the study region are represented as the restricted zone, and the alignment is not allowed to pass through this zone. The total area of these sensitive areas is about 25 percent of the study area. The objective function associated in the process of optimization of the horizontal alignment is to minimize the location dependent and length dependent cost.

\section{APPLICATION OF PPM}

The proposed model is used to find an optimum horizontal alignment between Angul and Talcher. The alignment is considered for two-lane two-way highways. Each lane is of $3.75 \mathrm{~m}$ with shoulder of $1.25 \mathrm{~m}$ on either side of the travelling lanes. Hence, the total width of the roadway is $10 \mathrm{~m}$. The proposed PPM depends on various input parameters to develop the horizontal alignment. These input parameters depend on the study region. For developing an optimal horizontal alignment it is important to select the input parameters properly. The subsequent section describes each input parameters.

\subsection{Selection of step size}

Step size helps to define the location of the optimum PIs, which are joined for the development of the horizontal alignment. It is decided based on the number and density of the restricted areas within the study area. The distance between any two points generated in the study area will be maintained within the given range of the step size. For the study area considered in this study, the step size of $800 \mathrm{~m}$ with a range of $650 \mathrm{~m}$ to $950 \mathrm{~m}$ is selected (Refer Fig. 3). This range is selected considering the minimum tangent length [23]. As the long straight section may generate driving fatigue, so, it is important to properly select the range for the placement of the PIs.

\subsection{Random point population}

The random point population plays an important role in the PPM. These random points (i.e., the possible PI's) are iteratively generated in the entire study area that helps to obtain an optimal horizontal alignment. By running the program for several times for different study area, it has been concluded that the number of random point's population size should vary from 100 to 150 times of minimum population. As it is known, that the shortest distance between any two points is the straight line i.e., Euclidean distance. So, the minimum number of points that needs to generate an alignment between any two points is defined by eqn (6):

$$
\text { Minimum Population }=\frac{\text { Euclidean Distance }}{S+150},
$$

where, $S$ is defined as step size and is determined from Fig. 3. The average step size value obtained for all the scenarios possessing different densities is different. Considering $300 \mathrm{~m}$ 


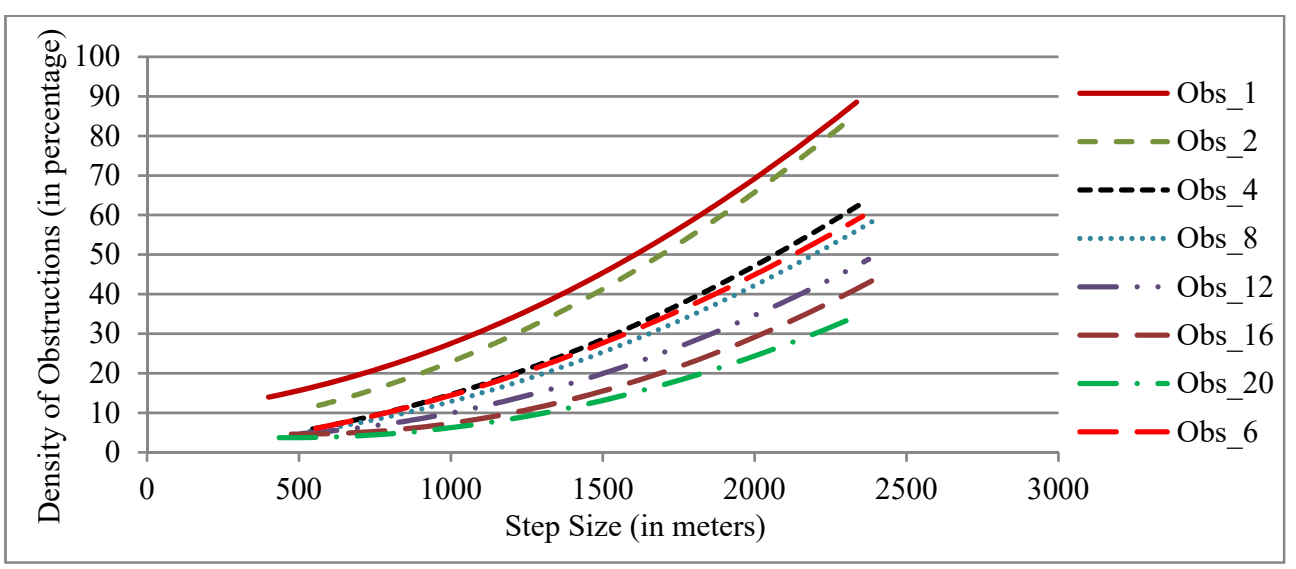

Figure 3: Step size calculation for the study area.

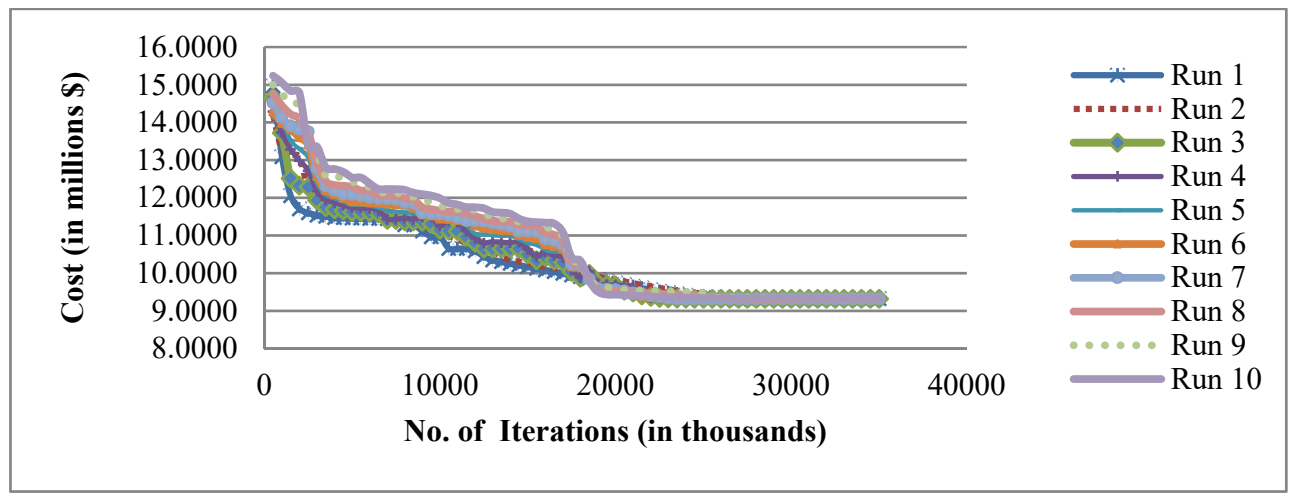

Figure 4: Cost of horizontal alignment generated for 10 runs.

as the minimum tangent length, as per the guidelines [1], the difference between the lower and the upper bound is considered as $300 \mathrm{~m}$, i.e., $\pm 150 \mathrm{~m}$ from the selected step size from Fig.3.

The random point population of 2500 is considered for this study. PPM is probabilistic and the result of the solution depends on the random population, so higher the population better is the result.

\subsection{Determination of number of iterations:}

As mentioned previously, the method generates a tree like path that iteratively increments between the two end points. It can be said that it controls the generation of the random population in the study area. Therefore, selection of optimal number of iteration is important in determining an optimal alignment. The selection of the number of the iteration depends upon the size of the study area. Larger is the size of the study area, higher is the number of iteration. However, the method checks the cost at every 5000 iterations, and if there is no improvement for 3 consecutive check, then the method terminates. These values are 
Table 2: Input design parameters for the problem.

\begin{tabular}{|c|c|c|}
\hline & Parameters & Values \\
\hline $\begin{array}{l}\text { Design } \\
\text { parameters }\end{array}$ & $\begin{array}{r}\text { Width of the road } \\
\text { Design Speed }\end{array}$ & $\begin{array}{r}10 \mathrm{~m} \text { (2-lanes, each } 3.75 \mathrm{~m} \text { and shoulder } \\
2.5 \mathrm{~m}) \\
100 \mathrm{~km} / \mathrm{hr}\end{array}$ \\
\hline $\begin{array}{l}\text { Constraint } \\
\text { parameters }\end{array}$ & $\begin{array}{r}\text { Coefficient of lateral } \\
\text { friction } \\
\text { Maximum Super elevation } \\
\text { Minimum curve radius }\end{array}$ & $\begin{array}{r}0.15 \\
4 \% \\
450 \mathrm{~m}\end{array}$ \\
\hline $\begin{array}{l}\text { Cost } \\
\text { parameters }\end{array}$ & $\begin{array}{r}\text { Price of road per } \mathrm{m}^{2} \\
\text { Price of impact area per } \mathrm{m}^{2}\end{array}$ & $\begin{array}{r}\$ 150 \\
\$ 50 \\
\end{array}$ \\
\hline
\end{tabular}

concluded by executing the program for 10 times, as it is a probabilistic method, and the trend of the cost at every 5000 iterations is observed as shown in Fig. 4.

The study area is considered to be a plain terrain. Table 2 shows the geometric parameters used in the study.

\section{RESULTS AND ANALYSIS}

Three numerical cases studies were considered to demonstrate the efficiency of the model. These three cases are briefly classified as:

a) PI's generated on regularly spaced orthogonal planes and joined with fixed radius curve.

b) Horizontal alignment with flexible PI's but joined with a constant curve radius; and

c) Flexible PI's with variable curve radius.

All of the experiments are carried out in a Dell workstation with an Intel(R) Core(TM) i53470 CPU@3.20 GHz (2 cores) processor. MATLAB2014b was used solve the Optimization problem. Case (a) is adopted by considering the generation of PI's by the existing methods. Case (b) is to make a comparison for adopting a curve with variable radius over constant value. In all the three cases, the study area and the number of iterations required to obtain the optimal result are same. Fig. 4 shows the resulting highway alignment obtained with all the three cases.

In case (a), the distance between the equally spaced eight orthogonal planes is $2.25 \mathrm{~km}$ (i.e., Euclidean distance, $18 \mathrm{~km}$ divided into eight equally spaced distance.). In this case the model searches for the PI in the consecutive orthogonal plane, where it can draw a direct connection avoiding the obstacle. The radius of the curve in this case is fixed to $450 \mathrm{~m}$. In case (b), the PIs of the alignment are not confined to any orthogonal planes, but the horizontal curves fitted between the tangential sections have a constant radius of $450 \mathrm{~m}$. The objective values obtained by solving all the three scenarios are presented in Table 3. This table states the cost obtained by generating the alignment on the same study area, with same parameters but by considering different models.

From the Table 3 it can be concluded that the alignment generated in case (c) possess minimum length as compared to that of case (a) and case (b). It is to be noted that the overall impact on land parcels reserved for environment has no impact in all the three cases, but still there is a huge difference in the alignment cost. In case (a), the model searches for the PIs only on the adjacent orthogonal planes, this restricts the search for an optimal alignment. Whereas, PPM would generate PIs near to the sensitive zone, without bounding it to any plane sections. This would help the PPM to bypass the sensitive zone with minimum distance compared to case (a), by decreasing the overall length of the alignment. 


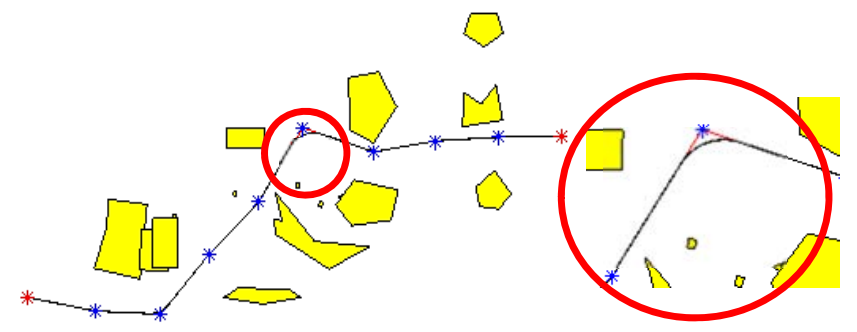

(a) PI's on regularly spaced orthogonal planes and constant curve radius.

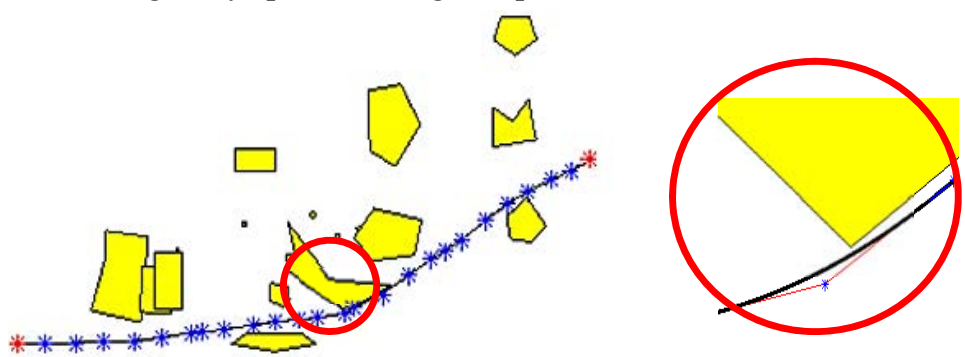

(b) Flexible PI's and constant curve radius.

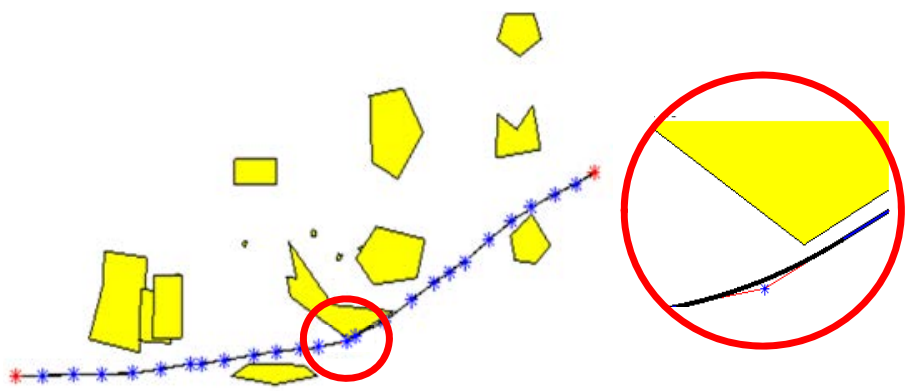

(c) Flexible PI's and variable curve radius.

Figure 5: Horizontal alignment for different cases.

Table 3: Objective values of representative horizontal alignment optimization solutions.

\begin{tabular}{|c|c|c|c|}
\hline Parameters & $\begin{array}{c}\text { Pl's on fixed orthogonal } \\
\text { planes and Constant } \\
\text { Curve Radius }\end{array}$ & $\begin{array}{c}\text { Flexible PI's and } \\
\text { Constant Curve } \\
\text { Radius }\end{array}$ & $\begin{array}{c}\text { Flexible PI's and } \\
\text { Variable Curve } \\
\text { Radius }\end{array}$ \\
\hline Length of road & $23.75 \mathrm{~km}$ & $22.58 \mathrm{~km}$ & $21.62 \mathrm{~km}$ \\
Environmental Impact & $0 \mathrm{sq} . \mathrm{m}$ & $0 \mathrm{sq} . \mathrm{m}$ & $0 \mathrm{sq} . \mathrm{m}$ \\
Total cost of road & $\$ 11.09 \mathrm{million}$ & $\$ 10.05 \mathrm{million}$ & $\$ 9.29 \mathrm{million}$ \\
Total Time taken & $790 \mathrm{~s}$ & $980 \mathrm{~s}$ & $1700 \mathrm{~s}$ \\
\hline
\end{tabular}

In cases (a) and (b) the radius of the curvature is constant i.e., the minimum curvature radius. With fixed radius, it is difficult to fit the curves in the real field, so it's better to adopt an appropriate radius as per the availability in the field. Fixed radius (i.e., minimum radius value) also increase the unwanted number of curves, which can be replaced by an appropriate 
or larger radius curve. This also affects the overall length of the alignment. PPM considers both maximum and minimum radius of curvature, and these radiuses are fitted as per the availability of the distance between the tangent sections, considering the design guidelines requirements. It is also easy to fit the curve in the hilly areas, or areas with complex environment with variable radius. However, there is a difference in computation time taken for development of the alignment by all the three cases. The execution time for case (c) is more as compared to the other two cases i.e., (a) and (b). This is because of the variable radius optimization process in stage two. In case (c), as the PIs are not confined to predefined orthogonal planes, so the computation time for finding the optimal PIs in the entire study area is more. Moreover, the execution time taken for fitting the curve by variable radius is more as compared to the fixed radius.

\section{DISCUSSION AND CONCLUSIONS}

The highway alignment optimization process optimizes horizontal alignment and also simultaneously minimizes the total cost of the generated alignment. In this process, the generated alignment satisfies code requirements as well as obligatory point requirements. The comparison of costs is shown in Table 3. PPM generates the horizontal alignment in two different stages. In first stage, an initial path is generated between the two desired points and in the second stage; the curve is fitted between the resulting tangents obtained from the PIs from the first stage. The model uses GIS maps to obtain the coordinates of the start and the end points. These coordinates information and the algorithm processes are computer coded in MATLAB. Due to its flexibility in PIs, this approach can be easily adopted to optimize alignments in highly irregular geographic spaces such as valleys in mountainous terrains and densely populated areas in plain terrains. Results obtained by the method for a small stretch of area are promising. The model can easily be extended for a large search area. The final solution is a highway alignment composed of line segments and horizontal curves in a similar manner as used by highway engineers in practice. The existing models develop alignment based on the PI's generated on particular plane and joined by a constant curve radius, reduces the flexibility of the alignment, which can lead to changes in the design stage. This paper calls for new techniques to overcome such limitations, and to bridge a gap between the planning and the design stage. This research can be extended in several directions in the future. It can be extended to incorporate it for the development of the vertical alignment. Three-dimensional alignment is also worth investigating in future studies.

\section{REFERENCES}

[1] Transportation Officials. A Policy on Geometric Design of Highways and Streets 2011. Washington, DC, 2011.

[2] Jha, M.K., Optimizing highway networks: A genetic algorithms and swarm intelligence based approach In Computing in Civil Engineering, pp. 76-89. ASCE, 2002.

[3] Jha, M.K., Criteria-based decision support system for selecting highway alignments. Journal of Transportation Engineering, 129(1), 33-41, 2003.

[4] Jha, M.K. \& Schonfeld, P., Integrating genetic algorithms and geographic information system to optimize highway alignments. Journal of the Transportation Research Board, 1719(1), pp. 233-240, 2000.

[5] Jong, J.C., Jha, M.K. \& Schonfeld, P., Preliminary highway design with genetic algorithms and geographic information systems. Computer-Aided Civil and Infrastructure Engineering, 15(4), pp. 261-271, 2000. 
[6] Shafahi, Y. \& Bagherian, M., A customized particle swarm method to solve highway alignment optimization problem. Computer-Aided Civil and Infrastructure Engineering, 28(1), pp. 52-67, 2013.

[7] Howard, B.E., Bramnick, Z. \& Shaw, J.F., Optimum Curvature Principle in Highway Highway Routing, pp. 236-241, 1969.

[8] Parker, N.A., Rural highway route corridor selection. Transportation Planning and Technology, 3(4), pp. 247-256, 1977.

[9] Kang, M.W. et al. Applicability of highway alignment optimization models. Transportation Research Part C. Emerging Technologies, 21(1), 2012.

[10] Mondal, S. et al. Optimizing horizontal alignment of roads in a specified corridor. Computers \& Operations Research, 64, pp. 130-138, 2015.

[11] Shaw, J.F. \& Howard, B.E. Expressway route optimization by OCP. Journal of Transportation Engineering, 108, 1982.

[12] Thomson, N.R. \& Sykes, J.F., Route selection through a dynamic ice field using the maximum principle. Transportation Research Part B: Methodological, 22(5), 1988.

[13] Wan, F., Introduction to the Calculus of Variations and its Applications. CRC Press, 1995.

[14] Trietsch, D., A family of methods for preliminary highway alignment. Transportation Science, 21(1), pp. 17-25, 1987.

[15] Turner, A.K. \& Miles, R.D., The GCARS system: A computer-assisted method of regional route location (No. 348), 1971.

[16] Athanassoulis, G.C. \& Calogero, V., Optimal location of a new highway from A to BA computer technique for route planning. In PTRC Seminar Proceedings on Cost Models \& Optimization in Highway, p. 9. 1973.

[17] Hogan, J.D., Experience with OPTLOC optimum location of highways by computer. PTRC Seminar Proceedings on Cost Models and Optimization in Highways (Session L10), London, 1973.

[18] Nicholson, A. J., Elms, D. G. \& Williman, A., A variational approach to optimal route location. Highway Engineer, 23(3), 1976.

[19] Tat, C.W. \& Tao, F., Using GIS and genetic algorithm in highway alignment optimization. In Intelligent Transportation Systems, 2003. Proceedings. 2003 IEEE 2, pp. 1563-1567,2003.

[20] Maji, A. \& Jha, M.K., Multi-objective highway alignment optimization using a genetic algorithm. Journal of Advanced Transportation, 43(4), pp. 481-504, 2009.

[21] Maji, A. \& Jha, M.K. A Multiobjective Analysis of Impacted Area of Environmentally Preserved Land and Alignment Cost for Sustainable Highway Infrastructure Design. Procedia-Social and Behavioral Sciences, 20, pp. 966-972, 2011.

[22] Lee, Y. et al., Optimization method for highway horizontal alignment design. Journal of Transportation Engineering, 135(4), pp. 217-224. 2009.

[23] Adiyatov, O. \& Varol, H.A., Rapidly-exploring random tree based memory efficient motion planning. Mechatronics and Automation (ICMA), 2013 IEEE International Conference, pp. 354-359, 2013.

[24] LaValle, S.M. \& Kuffner Jr, J.J., Rapidly-exploring Random Trees: Progress and Prospects, (2000).

[25] Lindemann, S.R. \& LaValle, S.M., Incrementally reducing dispersion by increasing Voronoi bias in RRTs. Robotics and Automation, 2004. Proceedings. ICRA'04. 2004 IEEE International Conference on IEEE 4, pp. 3251-3257, 2004. 